Discussion The COVID pandemic has a significant effect on sexual health care in a metropolitan region of the US; that effect is lasting, especially in areas of medium and high chlamydia rates. Health systems must consider how the pandemic has impacted care provided for all major public health problems, including STIs/HIV or risk worsening sexual health.

\section{P255 TROUBLING SILENCES: REFLECTING ON FINDINGS FROM AN INTEGRATIVE REVIEW ON ACCESS TO PREP}

${ }^{1} T$ Trombetta*, ${ }^{2}$ J Benito Tovillo, ${ }^{2} \mathrm{~V}$ Caine. ${ }^{1}$ Go Freddie, Edmonton, Canada; ${ }^{2}$ University of Alberta, Canada

10.1136/sextrans-2021-sti.330

Background In 2012, pre-exposure prophylaxis (PrEP) was approved as an HIV-prevention intervention. PrEP is a highly effective strategy for reducing the risk of HIV acquisition, particularly in populations at high risk of contracting the virus. In an integrative review of the literature, we explored barriers and facilitators to accessing PrEP and outlined potential interventions to mitigate access. In the integrative review, 48 studies were included. We also extracted data that provided information on potential interventions and recommendations that stakeholders and decision-makers can utilize to advance practice guidelines and health policies that will improve PrEP access among high-risk populations. In this paper, we reflect on the review findings and contemplate the silences that became visible when looking across all studies.

Methods We engaged in the process of reflexivity as we looked across the included studies. Throughout this process, we made notes, engaged in conversations, and consulted with others who work in the field. Results/

Conclusion We noted three significant areas of silence. One was a lack of intersectional analysis, which considers multiple minority-stress factors acting simultaneously. With little understanding of the complexities impacting understudied populations' intersectionalities, stakeholders and decision-makers lack not only formative contextual research, but also any effective implementable measure to increase PrEP uptake. The studies analyzed showed a lack of community-participatory research practice. At large, the studies found did not explore, nor perceive, communities at HIV risk as agents of their own health. Neither did they represent these communities as capable stakeholders and decision-makers in matters regarding sexual behavior and harm reduction. Peer-support involvement in public health measures to improve PrEP access has been scarce. Despite social connections and relationships representing efficient methods for PrEP awareness, education, and stigma reduction, peer involvement remains mostly unexplored in the literature in relation to PrEP access.

\section{P256 IDENTIFYING STI RISK GROUPS AMONG HETEROSEXUALS IN A COHORT STUDY BASED ON BEHAVIOURAL AND PSYCHOLOGICAL CHARACTERISTICS DURING THE COVID-19 PANDEMIC}

${ }^{1} \mathrm{D}$ van Wees ${ }^{*},{ }^{2} \mathrm{~N}$ Godijk, ${ }^{3} \mathrm{C}$ den Daas, ${ }^{2} \mathrm{M}$ Kretzschmar, ${ }^{1} \mathrm{~J}$ Heijne. ${ }^{1}$ Center for Infectious Diseases Control, National Institute for Public Health and the Environment, Bilthoven, The Netherlands; ${ }^{2}$ Julius Center for Health Sciences and Primary Care, University Medical Center Utrecht, Utrecht, The Netherlands; ${ }^{3}$ Aberdeen Health Psychology Group, Institute of Applied Health Sciences, Aberdeen, UK

10.1136/sextrans-2021-sti.331
Measures to reduce Coronavirus disease (COVID-19) transmission, including physical distancing, and downscaling of sexual health care impact behaviour and sexual health. We aimed to examine the impact of COVID-19 measures on sexual behaviour, and to characterize heterosexuals who were at high risk of acquiring sexually transmitted infection (STI) during the pandemic. A longitudinal cohort study (20162020) was conducted among Dutch heterosexual males and females aged 21-28 years in 2020. We used data on behavioural and psychological characteristics from: pre-lockdown (June-August 2019), lockdown (March-May 2020), and postlockdown (June-August 2020). Behaviour change was compared between subgroups identified with latent class analysis. Four latent classes were identified $(\mathrm{n}=239)$. Individuals in class 1 (48\% of study population) and $2(36 \%)$ were at low risk of acquiring STI pre-lockdown, during, and post-lockdown, and reported mostly steady partnerships. Individuals in class $3(9 \%)$ and $4(7 \%)$ reported multiple casual partners pre-lockdown, and class 4 continued having multiple partners during lockdown $(56 \%$ reported same/increased partner numbers compared to pre-lockdown versus $18 \%$ in class 3 ). Class 4 was characterized by less condom use, lower health goals, less positive STI/COVID-19 prevention attitudes, and higher impulsiveness compared to class 3. Post-lockdown, $36 \%$ in class 3 and $42 \%$ in class 4 reported same/increased partner numbers compared to pre-lockdown. Of individuals who wanted an STI test during or post-lockdown in class $3(57 \%)$ and $4(75 \%), 62 \%$ and $56 \%$ respectively did not get tested, mainly because they were not able to get an appointment. STI risk during the COVID-19 pandemic was low in most heterosexuals, but specific subgroups engaged in high-risk behaviour during lockdown and post-lockdown. During and after the COVID-19 pandemic, impulsive individuals with low health goals and less positive infection prevention attitudes should be prioritized for STI testing, and targeted with behavioural interventions tailored to these psychological characteristics.

\section{P257 ONLINE PARTNER SEEKING AS A SOCIAL PRACTICE: FINDINGS TO DEVELOP THE FOURTH NATIONAL SURVEY OF SEXUAL ATTITUDES AND LIFESTYLES}

${ }^{1} \mathrm{D}$ Reid*, ${ }^{1} \mathrm{C}$ Bonell, ${ }^{2} \mathrm{R}$ Lewis, ${ }^{3} \mathrm{~B}$ Hogan, ${ }^{2} \mathrm{~K}$ Mitchell, ${ }^{2} \mathrm{R}$ Bosó Pérez, ${ }^{4} \mathrm{~J}$ Gibbs, ${ }^{5} \mathrm{C}$ Smith, ${ }^{6} \mathrm{~F}$ Attwood, ${ }^{4} \mathrm{C}$ Mercer, ${ }^{4} \mathrm{P}$ Sonnenberg, ${ }^{1} \mathrm{~W}$ Macdowall, the Nastsal-4 Team. ${ }^{1}$ Department of Public Health, Environments and Society, London School of Hygiene and Tropical Medicine, London, UK; ${ }^{2} \mathrm{MRC/CSO}$ Social and Public Health Sciences Unit, University of Glasgow, Glasgow, UK; ${ }^{3}$ Oxford Internet Institute, University of Oxford, Oxford, UK; ${ }^{4}$ Institute for Global Health, University College London, London, UK; ${ }^{5}$ Northumbria University, Newcastle, UK; ${ }^{6}$ Independent Scholar, UK

\subsection{6/sextrans-2021-sti.332}

Background Rapid development and uptake of digital technologies have influenced sexual lives. As part of development research for the decennial British National Survey of Sexual Attitudes and Lifestyles (Natsal-4), we aimed to understand the practices of adults in Britain using digital technologies to meet sexual and romantic partners.

Methods We conducted 40 semi-structured interviews with adults in Britain on the role digital technologies played in their sexual lives. Here we draw on the accounts of 22 of those who had direct experience of online partner seeking. Informed by Social Practice Theory, we developed thematic 